\title{
PSYCHOLOGICA
}

\section{Interesses e competências percebidas em crianças do ensino básico}

Autor(es): $\quad$ David, Rute; Paixão, Maria Paula; Silva, José Tomás da

Publicado por: Imprensa da Universidade de Coimbra

URL

persistente:

URI:http://hdl.handle.net/10316.2/5462

DOI: $\quad$ DOI:http://dx.doi.org/10.14195/1647-8606_51_6

Accessed : $\quad$ 26-Apr-2023 05:42:30

A navegação consulta e descarregamento dos títulos inseridos nas Bibliotecas Digitais UC Digitalis, UC Pombalina e UC Impactum, pressupõem a aceitação plena e sem reservas dos Termos e Condições de Uso destas Bibliotecas Digitais, disponíveis em https://digitalis.uc.pt/pt-pt/termos.

Conforme exposto nos referidos Termos e Condições de Uso, o descarregamento de títulos de acesso restrito requer uma licença válida de autorização devendo o utilizador aceder ao(s) documento(s) a partir de um endereço de IP da instituição detentora da supramencionada licença.

Ao utilizador é apenas permitido o descarregamento para uso pessoal, pelo que o emprego do(s) título(s) descarregado(s) para outro fim, designadamente comercial, carece de autorização do respetivo autor ou editor da obra.

Na medida em que todas as obras da UC Digitalis se encontram protegidas pelo Código do Direito de Autor e Direitos Conexos e demais legislação aplicável, toda a cópia, parcial ou total, deste documento, nos casos em que é legalmente admitida, deverá conter ou fazer-se acompanhar por este aviso. 
NÚMERO 51

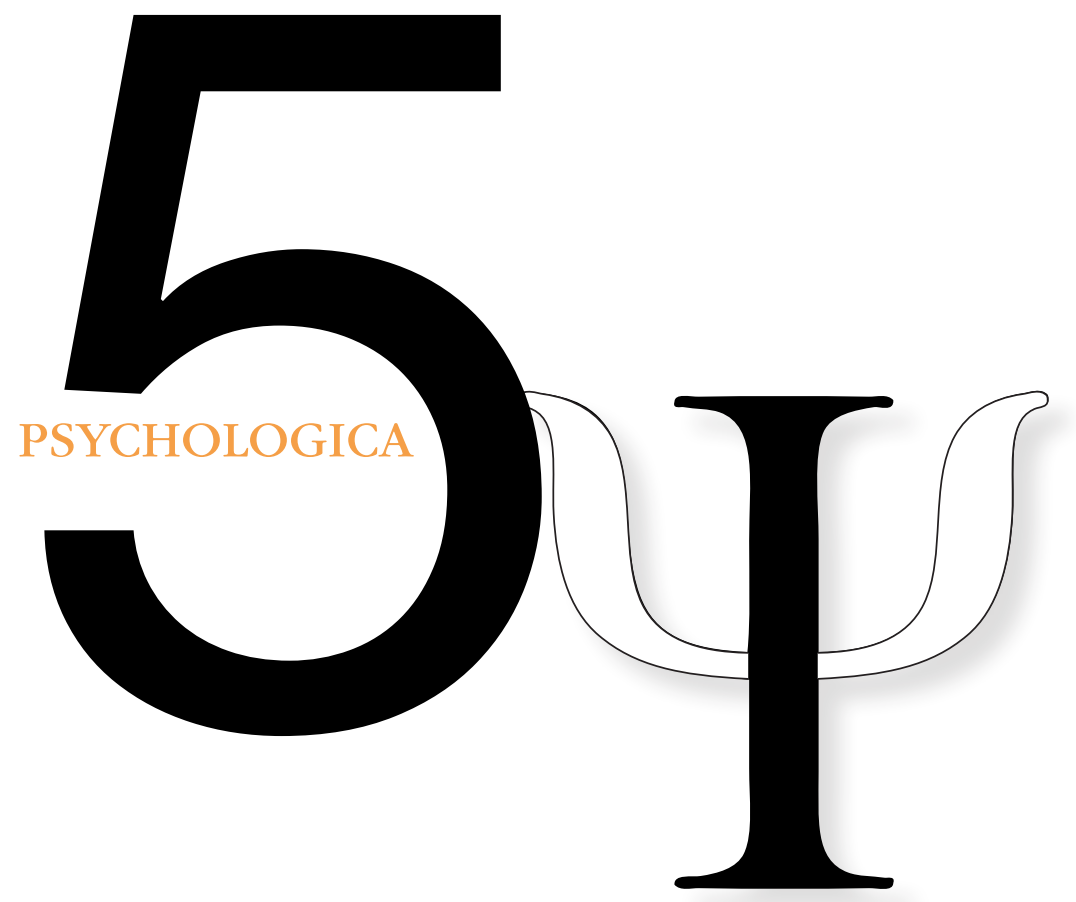

IMPRENSA DA UNIVERSIDADE DE COIMBRA

FACULDADE DE PSICOLOGIA E DE CIÊNCIAS DA EDUCAÇÃO DA UNIVERSIDADE DE COIMBRA 


\title{
Interesses e competências percebidas em crianças do ensino básico
}

\author{
Rute David', Maria Paula Paixão' \& José Tomás da Silva'
}

\begin{abstract}
Apesar de a infância ser considerada como a base do desenvolvimento vocacional, poucos são os estudos desenvolvidos nesta faixa etária acerca das variáveis implicadas e qual o papel que desempenham neste processo. Pretendendo colmatar algumas dessas lacunas procurámos explorar algumas características estruturais e correlacionais dos interesses e competências percebidas de crianças do ensino básico $\left(3^{\circ}, 6^{\circ}\right.$ e $9^{\circ}$ anos de escolaridade), recorrendo, para o efeito, à tradução e adaptação portuguesa do ICA-R de Tracey e Ward, que tem em consideração os seis tipos de personalidade enunciados por Holland (R-I-A-S-E-C).
\end{abstract}

PALAVRAS-CHAVE: Desenvolvimento vocacional; Infância; Competências percebidas; Interesses.

\section{Introdução}

Pesquisas recentes preocupam-se em analisar os aspectos desenvolvimentais da estrutura dos interesses, estando alguns destes trabalhos especialmente centrados na avaliação das crenças de competência, que se assumem como determinantes no desenvolvimento dos interesses (Lent, Tracey, Brown, Soresi, \& Nota, 2006).

Estando estes dois constructos relacionados, o estudo das percepções de competência tem sido concretizado por alguns autores de forma similar ao estudo dos interesses, usando a tipologia RIASEC de Holland (e.g., Betz, Harmon, \& Borgen, 1996; Holland, 1985), sendo que um número significativo de estudos incide na análise da similitude da estrutura entre interesses e percepções de competência (Boyle \& Fabris, 1992; Tracey, 1997).

Segundo Holland $(1973,1985)$, os interesses vocacionais podem ser categorizados em seis tipos: Realista, Investigador, Artístico, Social, Empreendedor e Convencional. Estes seis tipos RIASEC dispõem-se de forma hexagonal, retratando as

1 Faculdade de Psicologia e de Ciências da Educação, Universidade de Coimbra. 
semelhanças e diferenças entre os mesmos. Os tipos mais próximos (e.g. Social e Empreendedor) partilham alguns aspectos ao nivel dos interesses e características da personalidade, enquanto que para os tipos posicionados a maior distância e no vértice oposto no hexágono (e.g. Realista e Social) não existe sobreposição de interesses ou outro tipo de traços psicológicos (Hewig, 2003).

\subsection{Os Interesses Vocacionais}

A abordagem dos interesses sempre foi considerada pela orientação vocacional como uma "variável determinante da escolha de uma ocupação" (Pelietier, Noiseaux, \& Bujold, 1982, p. 190). Os interesses são, inclusive, considerados uns dos factores com maior impacto na Psicologia Vocacional (Betsworth \& Fouad, 1997), tendo sido vistos por Savickas (1999) como "um complexo esforço adaptativo de utilização do contexto pessoal para satisfação de necessidades e valores” (Savickas, 1999, cit in Leitão \& Miguel, 2001, p. 82).

Parece consensual considerar que, apesar do estabelecimento dos interesses ocorrer na infância, a sua estabilidade só ganha alguma consistência no início da idade adulta (Hartung, Porfeli, \& Vondracek, 2005).

As preferências profissionais das crianças do ensino básico parecem marcadas por uma certa estereotipia, em parte pela importância que a tipificação sexual tem nesta idade. No geral, os rapazes parecem eleger actividades ligadas ao movimento, à actividade física e imposição de ordens, enquanto as raparigas parecem preferir actividades mais sedentárias, ligadas a aspectos estéticos e aos serviços pessoais (idem, 2005).

Segundo Tracey (2000) a estrutura dos interesses das crianças vai mudando à medida que elas crescem. O autor refere, ainda, que a maior parte dos estudos acerca dos interesses das crianças se centra nas suas aspirações profissionais, numa idade em que as crianças ainda não têm um conhecimento muito alargado acerca das profissões. Como tal, sugere que o melhor modo de estudar os interesses das crianças é através do recurso às preferências que demonstram perante actividades concretas (o que raramente é feito). Para além disso, muitos estudos assumem antecipadamente que a estrutura circular da tipologia RIASEC de Holland (o modelo de estrutura de interesses mais prevalente na literatura, segundo o autor), está presente nas crianças, expectativa que não é inteiramente corroborada pelas pesquisas anteriores. Com efeito, os resultados de um estudo realizado com alunos dos $4^{\circ}-5^{\circ}$ anos, $6^{\circ}-8^{\circ}$ anos e estudantes universitários (Tracey \& Ward, 1998) indicaram que a estrutura circular da tipologia RIASEC estava associada positivamente à idade, ou seja, esta estrutura encontrava-se 
bem presente nos estudantes universitários, mas nos estudantes dos $6^{\circ}-8^{\circ}$ anos só o estava a um nível moderado, enquanto que em alunos mais novos $\left(4^{\circ}-5^{\circ}\right.$ anos) era pouco visível.

\subsection{Competências percebidas}

Segundo Tracey e Ward (1998), várias teorias têm associado causalmente o sentimento de competência ao desenvolvimento dos interesses (e.g. Super, 1990; Barak, 1981) e à escolha ocupacional. O sentimento de competência, mais comummente referido na literatura como auto-eficácia (pese embora as distinções que diversos autores têm efectuado entre estes dois conceitos, geralmente atribuindo ao conceito de auto-eficácia uma maior especificidade contextual), é um aspecto frequentemente enfatizado no desenvolvimento dos interesses, sobretudo com as crescentes a plicações da teoria sócio-cognitiva ao desenvolvimento de carreira (Bandura, 1977, 1982; Betz \& Hacket, 1981; Lent, Brown, \& Hackett, 1994).

A Teoria Sócio-Cognitiva de Carreira (Lent, Brown, \& Hackett, 2002), defende que os sujeitos mantêm o interesse numa actividade quando se percepcionam como competentes, ou auto-eficazes, e quando prevêem resultados positivos a partir do desempenho da mesma. Os interesses emergentes (juntamente com a auto-eficácia e as expectativas de sucesso), levam à formação de objectivos que sustentam ou aumentam o seu envolvimento nessa actividade. Por seu lado, estes objectivos aumentam a probabilidade de encetar e manter essa actividade. Os resultados obtidos com essa actividade fornecem um feedback importante que ajuda a solidificar ou a redefinir a auto-eficácia e expectativas de sucesso e, concomitantemente, os interesses (Lent et al., 2002).

As pessoas desenvolvem objectivos de escolha tomando em consideração as áreas escolares/profissionais que são congruentes com os seus interesses; no entanto, podem surgir limitações nas oportunidades relacionadas com o meio (variáveis externas) e até a personalidade (variáveis internas), que condicionam essas possíveis escolhas. Deste modo, os sujeitos vêm-se muitas vezes obrigados a comprometer os seus interesses e as suas escolhas são feitas, consequentemente, com base na acessibilidade a determinados empregos, nas suas crenças de auto-eficácia e nas suas expectativas de resultados (Taveira, 2004).

A percepção de competência e a auto-eficácia não se diferenciam facilmente. De acordo com Tracey e Ward (1998), a pesar de esta diferença ter sido identificada por alguns autores, como afirmámos anteriormente, na verdade não há um consenso sobre esta questão e, em última instância, poderíamos acrescentar que o diferendo pode ser resolvido através de estudos empíricos contemplando a avaliação 
simultânea dos dois construtos. Alguns investigadores concebem as percepções de competência como indicadores de auto-eficácia (e.g. Betz, Harmon, \& Borgen, 1996), enquanto outros as conceptualizam como distintas (e.g. Lopez, Lent, Brown, \& Gore, 1997). Lent, Tracey, Brown, Soresi e Nota (2006) realçam que a avaliação das crenças de auto-eficácia implica julgamentos acerca da capacidade prospectiva de organizar e executar acções mais complexas e abstractas, enquanto que as crenças de competência, por serem de ordem mais concreta, são relativamente mais fáceis de aceder pelas crianças, uma vez que estas têm apenas de se auto-avaliar (se faz bem ou mal) perante tarefas simples. Daí que, segundo os autores, as crenças de competência sejam uma boa analogia desenvolvimental da auto-eficácia, dado que podem facultar indícios acerca do desenvolvimento das crenças de auto-eficácia e seu relacionamento com os interesses nas crianças mais novas.

Segundo Holub (2005) a competência percebida é um componente do auto-conceito e reporta-se a uma crença individual acerca das próprias capacidades. De acordo com a autora, a competência percebida destaca-se do vasto leque das restantes auto-percepções por várias razões: (1) proeminência nos auto-relatos das crianças de referências às suas competências percebidas; (2) é um dos aspectos das auto-percepções das crianças facilmente mensurável e que, por outro lado, podem ser facilmente completadas por outros observadores (e.g. pais e professores); (3) e tal, como as competências reais, as competências percebidas mudam ao longo do tempo, à medida que as crianças adquirem mais competências cognitivas, sociais e físicas, se tornam mais conscientes das mesmas e começam a agir de modo mais independente.

As crianças mais novas tendem a sobrevalorizar as suas percepções de competência (Tracey \& Ward, 1998), mesmo em situação de fracasso iminente (Parsons $\&$ Ruble, 1977), ou após experienciarem o fracasso (Dweck \& Elliott, 1983). Estas auto-percepções positivas (viés optimista) durante os anos pré-escolares e primeiros anos de escolaridade são bastante benéficas, na medida em que as percepções de competência mantêm as crianças motivadas para iniciar e prosseguir com tarefas dificeis. As percepções elevadas de competência a nível académico têm sido igualmente relacionadas com o gosto pela escola e pela aprendizagem. As relações entre realização escolar, persistência da motivação e competência percebida parecem manter-se durante os anos escolares (Holub, 2005). Todavia, perto do final do $1^{\circ}$ ciclo do Ensino Básico estas pontuações tendem a diminuir, uma vez que as crianças começam a discriminar as competências por área e a ter em conta as competências dos outros, o que conduz a uma avaliação mais realista destas variáveis pessoais (Tracey \& Ward, 1998). Sabe-se também que as percepções que as crianças têm acerca de serem efectivamente responsáveis pelo sucesso ou insucesso das suas tentativas constituem um dos factores mais importantes para aprendizagens futuras e para a sua auto-estima (Sollerhed, Apitzsch, Råstam, \& Ejlertsson, 2008). 
Da revisão de estudos que realizámos pode concluir-se, desde logo, que as percepções de competência/auto-eficácia desempenham um papel assaz relevante no desenvolvimento vocacional das crianças. Paradoxalmente, embora vários autores influentes tenham reconhecido, nos seus trabalhos e posições teóricas, a necessidade de se estudar de forma mais sistemática e profunda as variáveis implicadas no processo de desenvolvimento vocacional durante o período da infância, a realidade porém demonstra que esse desejo não foi suficientemente cumprido. Em Portugal, apesar dos trabalhos já iniciados (e.g. Taveira, 2004), o panorama não difere do cenário internacional já referido. Urge, pois, examinar empiricamente em populações nacionais o processo de formação dos interesses vocacionais, o papel que diversas variáveis desempenham nesse processo, designadamente as variáveis pessoais relativas às percepções de competência ou de auto-eficácia.

O presente estudo procura colmatar algumas das lacunas do nosso conhecimento sobre a construção da carreira na infância. Especificamente, com este primeiro trabalho desejamos explorar algumas das características estruturais e correlacionais ao nível dos interesses e das competências percebidas em crianças do ensino básico, tendo em consideração os 6 tipos de personalidade vocacional enunciados por Holland: Realista, Investigador, Artístico, Social, Empreendedor e Social (RIA-SEC). Uma vez que até ao momento não há qualquer instrumento de avaliação psicológica dos construtos acima referenciados, constitui um importante objectivo deste estudo proceder à adaptação da versão Portuguesa do ICA-R de Tracey e Ward (1998), para a língua Portuguesa e obter os primeiros dados psicométricos sobre os resultados recolhidos em alunos do ensino básico.

\section{Método}

\subsection{Participantes}

A amostra deste estudo é composta por 383 sujeitos, alunos dos $3^{\circ}, 6^{\circ}$ e $9^{\circ}$ anos de escolaridade, sendo 218 alunos do sexo feminino (56,9\%) e 165 do sexo masculino (43,1\%). A grande maioria (79,9\%) é oriunda de áreas predominantemente urbanas (cf. Classificação do Instituto Nacional de Estatística de 1998 da Divisão Territorial Nacional: Tipologia de Área Urbana) e $64 \%$ de nível sócio-económico baixo (de acordo com a classificação de Simões, 1994).

Na totalidade da amostra, 25,6\% dos alunos já reprovaram de ano, sendo, no geral, muito aproximadas as percentagens de reprovação nos rapazes $(25,5 \%)$ e nas raparigas (25,7\%). A percentagem mais elevada de alunos com reprovações encontra-se no grupo dos alunos do $9^{\circ}$ ano de escolaridade $(35,1 \%)$, dado que é 
esperado, uma vez que este é o nível escolar mais avançado da nossa amostra e nele se concentram todas as reprovações escolares que os sujeitos tiveram ao longo da sua escolaridade até ao momento de aplicação dos questionários.

\subsection{Instrumentos}

No presente estudo foi utilizada a escala ICA-R (Inventory of Children's Activities - Revised) de Tracey e Ward (1998). O recurso a esta escala apoiou-se no facto de, por um lado, permitir o estudo dos interesses e das competências percebidas, um aspecto fundamental para o objectivo de averiguarmos quais as aspirações e relações que os alunos têm com a escolaridade e, por outro, porque é um dos poucos instrumentos, no domínio do comportamento vocacional, que se pode administrar a partir do $1^{\circ}$ ciclo do Ensino Básico.

Após contactarmos o primeiro autor da escala (Tracey) e de nos ter sido concedida a autorização para a utilização do inventário em Portugal, o ICA-R foi traduzido para Português. Esta versão foi de seguida retrovertida do Português para Inglês com o propósito de atestarmos a comparabilidade semântica de ambas as versões e a sua correspondência com a escala original. Este processo contou com a colaboração de um profissional Português na área da Orientação Escolar e Profissional e que está emigrado nos Estados Unidos da América. A versão que resultou deste procedimento foi administrada numa pequena amostra de alunos do $1^{\circ}$ ciclo do Ensino Básico, com a intenção de detectar possíveis dificuldades de compreensão das actividades descritas na escala (itens), assim como de obtermos uma estimativa do tempo médio necessário para o seu preenchimento.

A escala é composta por duas secções cada uma com 30 actividades que as crianças habitualmente fazem no seu quotidiano ( 5 por cada tipo da tipologia RIASEC de Holland). Na primeira secção solicita-se que assinalem numa escala de 1 ("Não gosto mesmo nada") a 5 ("Gosto muito") o quanto gostam da actividade apresentada. Na segunda secção pergunta-se em que medida se percebem como capazes de desempenharem as actividades enunciadas ( 1 = muito mau/ muito má; 5 = muito bom/muito boa). Os resultados da tipologia RIASEC, tanto ao nível dos interesses ( $1^{\mathrm{a}}$ secção) como das competências percebidas ( $2^{\mathrm{a}}$ secção), são calculados determinando a média aritmética das respostas aos 5 itens de cada uma das 6 sub-escalas.

Os autores do instrumento consideram, como já afirmámos anteriormente, ser mais apropriado o recurso a actividades do que aos títulos ocupacionais (e.g., bombeiro/a, advogado/a), uma vez que as crianças (principalmente as mais novas) ainda têm algumas limitações ao nível do conhecimento das profissões. 


\subsection{Análise de dados}

Para examinarmos as respostas nas múltiplas variáveis observadas recorremos a várias técnicas estatísticas descritivas e inferenciais. Em primeiro lugar, calculámos estatísticas descritivas (médias e desvios-padrão) para cada uma das sub-escalas de interesses e de competências percebidas, tomando em consideração o ano de escolaridade dos participantes. Uma vez que este é o primeiro estudo realizado com a versão Portuguesa do ICA-R procedemos, em segundo lugar, ao cálculo das estimativas de precisão (consistência interna) dos resultados de cada uma das escalas. Uma terceira análise, de tipo inferencial, visou determinar se as médias observadas para cada um dos tipos RIASEC (interesses e competências) diferiam estatisticamente $(p<0,05)$ em função dos níveis de escolaridade das crianças. Para testarmos esta hipótese foi inicialmente efectuada uma ANOVA unifactorial, completada posteriormente com testes a posteriori (Bonferroni) para podermos localizar os pares de médias realmente distintos. Uma quarta análise incidiu sobre a matriz de intercorrelações calculada, do mesmo modo, para cada um dos grupos de escolaridade considerados neste trabalho. Considerando que o presente estudo se insere numa secção especial sobre o conceito de auto-eficácia, apenas procedemos ao exame das correlações na dimensão referente às competências percebidas (vide David, 2007, para uma análise similar do padrão das relações entre os tipos de interesses vocacionais). Finalmente, procedemos a uma análise exploratória da hipótese correspondente à organização estrutural em forma circular dos seis tipos de competências percebidas.

Há várias opções técnicas disponiveis para avaliar a presença de modelos estruturais tipo circular nos dados. Entre os exemplos de abordagens exploratórias mais comummente utilizadas (e.g., Tracey, 2000) estão a análise factorial comum, a análise de componentes principais (PCA) e o escalonamento multidimensional (MDS). Esta última técnica, em particular, tem sido especialmente usada na literatura da Psicologia Vocacional para examinar a hipótese estrutural proposta por Holland (e.g., 1973), a respeito da organização das preferências vocacionais de tipo Realista, Investigador, Artístico, Social, Empreendedor e Convencional (RIASEC), na forma de um hexágono (evidentemente, segundo Guttman [veja-se Tracey, 200o], o modelo hexagonal é um caso especial do modelo circular, i.e., um modelo dito "circulant" que se define pelo igual espaçamento das variáveis em torno de um círculo).

O escalonamento multidimensional (MDS, Multidimensional scaling) abrange uma numerosa família de modelos que foram concebidos para "analisar dados de tipo distâncias, designados por dados de dissemelhança, ou dados que indicam o grau de dissemelhança (ou semelhança) entre duas coisas" (Young \& Harris, 2007, p. 336). Mais especificamente, segundo Carroll e Arabie (1980), "definido de forma lata, o escalonamento multidimensional inclui uma família de modelos geométricos 
para a representação multidimensional de dados e um correspondente conjunto de métodos para ajustar esses modelos aos dados propriamente ditos" (p. 608). Uma característica comum desta classe de modelos, como referem Everitt e Dunn (2001), consiste no facto de todos eles procurarem "representar a matriz de proximidade através de um modelo ou mapa geométrico simples” (p. 93).

Neste estudo o procedimento ALSCAL (Takane, Young, \& de Leeuw, 1977), tal como está implementado no programa SPSS Statistics, Inc. (release 17.0), foi o algoritmo usado para realizar as análises MDS nas três sub-amostras observadas $\left(3^{\circ}, 6^{\circ}\right.$ e $9^{\circ}$ anos de escolaridade). Mais precisamente, usámos o modelo MDS clássico não métrico (adequado para dados medidos numa escala ordinal), aplicando-o às distâncias (euclidianas) criadas pelo programa a partir dos valores médios obtidos pelos participantes nas seis variáveis de competências derivadas da tipologia de Holland. Vários aspectos serão considerados na interpretação dos resultados, designadamente o número de iterações necessárias para o programa alcançar uma solução e vários indicadores do grau de ajustamento das distâncias observadas entre os pares de objectos (variáveis) e as estimadas pelo computador (disparidades) -S-Stress (fórmula 1 de Young), Stress (fórmula 1 de Kruskal) e o coeficiente de determinação, $R^{2}$. Para ambas as fórmulas de Stress os valores podem localizar-se num intervalo entre o e 1, com valores próximos de zero representando um melhor ajuste. O quadrado do coeficiente de correlação múltiplo, como é habitual, varia entre o e 1, todavia, neste caso valores mais elevados indicam que uma maior proporção de variância das disparidades (i.e., dos dados escalonados de forma óptima) é explicada pelo procedimento MDS (Hair, Tatham, Anderson, \& Black, 1998). De particular interesse para avaliarmos a hipótese relativa à presença do padrão RIASEC na matriz observada são os mapas espaciais/perceptuais produzidos para cada amostra. Finalmente, não constituindo a questão relativa ao número de dimensões um aspecto fulcral da presente investigação, decidimos, após uma revisão da literatura, especificar duas dimensões como sendo o número suficiente de eixos para localizar um ponto na configuração MDS.

Todas as análises referidas foram executadas com recurso ao programa SPSS para Windows (versão 17.0).

\section{Resultados}

Em primeiro lugar apresentamos as médias e os desvios-padrão obtidos ao nível dos interesses e das competências percebidas por nível de escolaridade (vide Quadro 1). As médias aritméticas, quer nos interesses, quer nas competências percebidas, localizam-se em todos os casos acima do ponto médio natural da escala de avaliação usada no instrumento (escala com 5 pontos). Os valores médios mais altos 
correspondem, em ambos os casos, aos tipos Social e Artístico, enquanto que a média mais baixa diz respeito aos interesses e competências de tipo Realista ( $M$ $=3,72$ e $M=3,80$, respectivamente). As respostas apresentam valores de dispersão inferiores a um ponto, com o valor mínimo $(D P=0,48)$ correspondendo ao tipo Artístico (interesses, $3^{\circ}$ ano de escolaridade) e o valor máximo (DP $=0,77$ ) ao tipo Realista (interesses, $6^{\circ}$ ano; competências, $9^{\circ}$ ano).

Quadro 1. Estatísticas descritivas nos interesses nas competências percebidas e comparação das diferenças nas médias por ano de escolaridade

\begin{tabular}{|c|c|c|c|c|c|c|c|c|c|}
\hline \multirow[b]{2}{*}{ Escala } & \multicolumn{2}{|c|}{$\begin{array}{c}3^{\circ} \text { ano } \\
(n=90)\end{array}$} & \multicolumn{2}{|c|}{$\begin{array}{c}6^{\circ} \text { ano } \\
(n=142)\end{array}$} & \multicolumn{2}{|c|}{$\begin{array}{c}9^{\circ} \text { ano } \\
(n=151)\end{array}$} & \multirow[t]{2}{*}{$F$} & \multirow[t]{2}{*}{$p$} & \multirow{2}{*}{$\begin{array}{c}\text { Teste } \\
\text { Post-Hoc }\end{array}$} \\
\hline & $M$ & DP & $M$ & $\mathrm{DP}$ & $M$ & DP & & & \\
\hline \multicolumn{10}{|l|}{ Interesses } \\
\hline Realista & 3,72 & 0,71 & 3,26 & 0,77 & 2,77 & 0,76 & 47,049 & 0,000 & $3^{\circ}>6^{\circ}>9^{\circ}$ \\
\hline Investigador & 4,29 & 0,53 & 3,71 & 0,60 & 3,36 & 0,67 & 65,581 & 0,000 & $3^{\circ}>6^{\circ}>9^{\circ}$ \\
\hline Artístico & 4,44 & 0,48 & 3,94 & 0,62 & 3,68 & 0,59 & 48,914 & 0,000 & $3^{\circ}>6^{\circ}>9^{\circ}$ \\
\hline Social & 4,51 & 0,50 & 4,00 & 0,60 & 3,91 & 0,64 & 33,051 & 0,000 & $3^{\circ}>6^{\circ}>9^{\circ}$ \\
\hline Empreendedor & 4,07 & 0,63 & 3,68 & 0,57 & 3,47 & 0,61 & 27,938 & 0,000 & $3^{\circ}>6^{\circ}>9^{\circ}$ \\
\hline Convencional & 4,16 & 0,58 & 3,59 & 0,58 & 3,25 & 0,64 & 64,662 & 0,000 & $3^{\circ}>6^{\circ}>9^{\circ}$ \\
\hline \multicolumn{10}{|l|}{ Competências } \\
\hline Realista & 3,80 & 0,70 & 3,28 & 0,76 & 2,82 & 0,77 & 49,728 & 0,000 & $3>{ }^{\circ} 6^{\circ}>9^{\circ}$ \\
\hline Investigador & 4,28 & 0,63 & 3,67 & 0,61 & 3,37 & 0,71 & 53,503 & 0,000 & $3^{\circ}>6^{\circ}>9^{\circ}$ \\
\hline Artístico & 4,46 & 0,51 & 3,92 & 0,57 & 3,63 & 0,63 & 57,952 & 0,000 & $3^{\circ}>6^{\circ}>9^{\circ}$ \\
\hline Social & 4,31 & 0,62 & 3,89 & 0,60 & 3,79 & 0,63 & 20,678 & 0,000 & $3^{\circ}>6^{\circ}>9^{\circ}$ \\
\hline Empreendedor & 4,09 & 0,63 & 3,58 & 0,61 & 3,42 & 0,59 & 36,216 & 0,000 & $3^{\circ}>6^{\circ}>9^{\circ}$ \\
\hline Convencional & 4,18 & 0,66 & 3,63 & 0,60 & 3,33 & 0,66 & 50,055 & 0,000 & $3^{\circ}>6^{\circ}>9^{\circ}$ \\
\hline
\end{tabular}

${ }^{*}$ Teste Post-Hoc (Bonferroni)

Comparando as médias obtidas por ano de escolaridade, verifica-se a existência de diferenças estatisticamente significativas $(p<0,05)$ em todos as dimensões, sendo a direcção destas diferenças sempre constante, ou seja, é a amostra do $3^{\circ}$ ano de escolaridade que obtém resultados mais elevados, seguida da do $6^{\circ}$ ano, sendo na amostra de alunos do $9^{\circ}$ ano que se registam valores mais baixos. Este decréscimo que se verifica à medida que a idade aumenta (tanto nos interesses como nas competências percebidas) também foi obtido nos estudos de Tracey e Ward (1998), tendência que foi justificada pelos autores como estando relacionada com o aumento do realismo na escolha ocupacional e nas percepções de competência nas crianças mais velhas.

O grau de precisão (consistência interna ou homogeneidade) das respostas foi calculado com base no coeficiente alfa de Cronbach. No conjunto da amostra (após agregação das três amostras de respondentes) os resultados no ICA-R apresentam, 
ao nível dos interesses, uma consistência interna de 0,78 para o tipo Realista, 0,72 para o Investigador, o,63 para o Artístico, o,60 para o Empreendedor e o,69 para o Convencional. Ao nível das competências percebidas os valores são ligeiramente superiores: 0,80 para o tipo Realista, 0,78 para o Investigador, 0,66 para o Artístico, o,75 para o Social, o,67 para o Empreendedor e o,74 para o Convencional. Os valores alfa de Cronbach das respostas desagregadas em função de cada uma das amostras observadas foram igualmente calculados. Podemos verificar, pela análise do Quadro 2, que a consistência interna tende a aumentar com a idade e com a frequência de níveis e escolaridade mais avançados.

Os resultados sugerem uma consistência interna do instrumento razoável e são consonantes com os valores relativos à consistência do ICA-R encontrados pelos autores da escala (Tracey \& Ward, 1998).

Quadro 2. Consistência interna do ICA-R por ano de escolaridade

\begin{tabular}{|c|c|c|c|c|c|c|}
\hline & $R$ & $\mathrm{I}$ & $A$ & $S$ & $\mathrm{E}$ & C \\
\hline \multicolumn{7}{|c|}{$3^{\circ}$ ano $(n=90)$} \\
\hline \multicolumn{7}{|l|}{ Interesses } \\
\hline Alfa de Cronbach & 0,61 & 0,55 & 0,48 & 0,67 & 0,46 & 0,58 \\
\hline \multicolumn{7}{|l|}{ Competências } \\
\hline Alfa de Cronbach & 0,60 & 0,73 & 0,54 & 0,77 & 0,55 & 0,70 \\
\hline \multicolumn{7}{|c|}{$6^{\circ}$ ano $(n=142)$} \\
\hline \multicolumn{7}{|l|}{ Interesses } \\
\hline Alfa de Cronbach & 0,73 & 0,63 & 0,63 & 0,70 & 0,60 & 0,59 \\
\hline \multicolumn{7}{|l|}{ Competências } \\
\hline Alfa de Cronbach & 0,77 & 0,66 & 0,54 & 0,74 & 0,67 & 0,66 \\
\hline \multicolumn{7}{|c|}{$9^{\circ}$ ano $(n=151)$} \\
\hline \multicolumn{7}{|l|}{ Interesses } \\
\hline Alfa de Cronbach & 0,76 & 0,68 & 0,54 & 0,67 & 0,56 & 0,59 \\
\hline \multicolumn{7}{|l|}{ Competências } \\
\hline Alfa de Cronbach & 0,82 & 0,77 & 0,60 & 0,70 & 0,60 & 0,66 \\
\hline
\end{tabular}

Com efeito, na sua amostra de alunos do ensino básico do segundo ciclo-elementary school - (134 alunos dos $4^{\circ}$ e $5^{\circ}$ anos), ao nível dos interesses foi encontrada uma consistência de 0,76 para o tipo Realista (o,61 na nossa amostra do $3^{\circ}$ ano), 0,52 para o Investigador ( 0,55 na nossa amostra), 0,58 para o Artístico (o,48 na nossa amostra), o,73 para o Social (o,67 na nossa amostra), 0,38 para o Empreendedor (o,46 na nossa amostra) e o,68 para o Convencional (0,58 na nossa amostra). Ao nivel das competências percebidas os valores registados foram de 0,75 para o tipo Realista (o,6o na nossa amostra), 0,64 para o Investigador (0,73 na nossa amostra), 0,52 para o Artístico (0,54 na nossa amostra), 0,75 para o Social (0,77 na nossa amostra), 0,57 para o Empreendedor (0,55 na nossa amostra) e 0,70 para o Convencional (o,70 na nossa amostra). De notar que a nossa amostra do $3^{\circ}$ ano 
tinha apenas 90 sujeitos e que os valores de consistência interna poderiam ser superiores se a amostra fosse maior.

$\mathrm{Na}$ amostra do $3^{\circ}$ ciclo do ensino básico - middle school - $\left(607\right.$ alunos do $6^{\circ}$, $7^{\circ}$ e $8^{\circ}$ anos de escolaridade) os valores registados por Tracey e Ward (1998) ao nível dos interesses foram de 0,75 para o tipo Realista (0,73 na nossa amostra do $6^{\circ}$ ano), o,63 para o Investigador (o,63 na nossa amostra), o,47 para o Artístico (o,63 na nossa amostra), 0,70 para o Social (o,70 na nossa amostra), o,41 para o Empreendedor (o,6o na nossa amostra) e 0,72 para o Convencional (0,59 na nossa amostra). Ao nivel das competências percebidas a consistência interna foi de 0,76 para o tipo Realista (0,77 na nossa amostra), o,65 para o Investigador (o,66 na nossa amostra), 0,52 para o Artístico (o,54 na nossa amostra), 0,76 para o Social (o,74 na nossa amostra), o,56 para o Empreendedor (o,67 na nossa amostra) e 0,74 para o Convencional (o,66 na nossa amostra).

Analisando as correlações entre as competências percebidas relativamente aos tipos RIASEC no total da amostra (Quadro 3) concluímos que em todos os tipos existem correlações bastante elevadas $(p<0,01)$.

Quadro 3. Correlações das competências percebidas entre os tipos RIASEC obtidas na amostra total

\begin{tabular}{|c|c|c|c|c|c|c|c|}
\hline & & \multicolumn{6}{|c|}{ Competências } \\
\hline & & $\mathrm{R}$ & I & $A$ & $S$ & E & $C$ \\
\hline \multirow{6}{*}{ 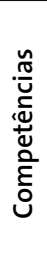 } & $\mathrm{R}$ & 1 & $0,583^{* *}$ & $0,391^{* *}$ & $0,241^{* *}$ & $0,465^{* *}$ & $0,517^{* *}$ \\
\hline & 1 & $0,583^{* *}$ & 1 & $0,596^{* *}$ & $0,502^{* *}$ & $0,469^{* *}$ & $0,586^{* *}$ \\
\hline & A & $0,391^{* *}$ & $0,596^{* *}$ & 1 & $0,612^{* *}$ & $0,546^{* *}$ & $0,570^{* *}$ \\
\hline & $\mathrm{S}$ & $0,241^{* *}$ & $0,502^{* *}$ & $0,612^{* *}$ & 1 & $0,527^{* *}$ & $0,542^{* *}$ \\
\hline & $\mathrm{E}$ & $0,465^{* *}$ & $0,469^{* *}$ & $0,546^{* *}$ & $0,527^{* *}$ & 1 & $0,532^{* *}$ \\
\hline & $C$ & $0,517^{* *}$ & $0,586^{* *}$ & $0,570^{* *}$ & $0,542^{* *}$ & $0,532^{* *}$ & 1 \\
\hline
\end{tabular}

${ }^{*} \mathrm{p}<0,05^{* *} \mathrm{p}<0,01$

No total da amostra, as competências de tipo Realista estão mais fortemente associados às competências de tipo Investigador; as de tipo Investigador às de tipo Artístico; as competências de tipo Artístico mais associadas às competências de tipo Social; as de tipo Empreendedor às de tipo Artístico e; as competências de tipo Convencional às competências de tipo Investigador.

Relativamente ao $3^{\circ}$ ano (Quadro 4) a análise dos dados relativamente às competências de tipo Realista indicam-nos que este tipo está mais fortemente associado ao tipo Investigador; o tipo Investigador mais associado ao tipo Social; as competências de tipo Artístico às de tipo Social; as de tipo Empreendedor às competências de tipo Artístico e as de tipo Convencional às competências de tipo Investigador. 
Quadro 4. Correlações das competências percebidas entre os tipos RIASEC obtidas no $3^{\circ}$ ano

84

\begin{tabular}{|c|c|c|c|c|c|c|c|}
\hline \multirow{2}{*}{\multicolumn{2}{|c|}{$3^{\circ}$ ano }} & \multicolumn{6}{|c|}{ Competências } \\
\hline & & $\mathrm{R}$ & I & $A$ & $S$ & $\mathrm{E}$ & C \\
\hline \multirow{6}{*}{ 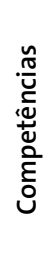 } & $\mathrm{R}$ & 1 & $0,512^{* *}$ & $0,292^{* *}$ & $0,327^{* *}$ & $0,456^{* *}$ & $0,452^{* *}$ \\
\hline & 1 & $0,512^{* *}$ & 1 & $0,474^{* *}$ & $0,541^{* *}$ & $0,404^{* *}$ & $0,701^{* *}$ \\
\hline & A & $0,292^{* *}$ & $0,474^{* *}$ & 1 & $0,679^{* *}$ & $0,567^{* *}$ & $0,601^{* *}$ \\
\hline & $\mathrm{S}$ & $0,327^{* *}$ & $0,541^{* *}$ & $0,679^{* *}$ & 1 & $0,489^{* *}$ & $0,627^{* *}$ \\
\hline & $E$ & $0,456^{* *}$ & $0,404^{* *}$ & $0,567^{* *}$ & $0,489^{* *}$ & 1 & $0,451^{* *}$ \\
\hline & C & $0,452^{* *}$ & $0,701^{* *}$ & $0,601^{* *}$ & $0,627^{* *}$ & $0,451^{* *}$ & 1 \\
\hline
\end{tabular}

${ }^{*} p<0,05{ }^{* *} p<0,01$

No $6^{\circ}$ ano (Quadro 5) as competências de tipo Realista estão mais fortemente associadas às competências de tipo Investigador; as de tipo Investigador às de tipo Artístico; as de tipo Artístico às de tipo Investigador e Social; as de tipo Social às de tipo Artístico; as de tipo Empreendedor às de tipo Social e Convencional e as de tipo Convencional às de tipo Investigador.

No $9^{\circ}$ ano (Quadro 6), as competências de tipo Realista estão mais fortemente associadas ao tipo Investigador; as de tipo Investigador às de tipo Realista e Artístico; as de tipo Artístico às de tipo Social; as de tipo Empreendedor às de tipo Artístico e Social e as de tipo Convencional às de tipo Social.

Quadro 5. Correlações das competências percebidas entre os tipos RIASEC obtidas no $6^{\circ}$ ano

\begin{tabular}{|c|c|c|c|c|c|c|c|}
\hline \multirow{2}{*}{\multicolumn{2}{|c|}{$6^{\circ}$ ano }} & \multicolumn{6}{|c|}{ Competências } \\
\hline & & $\mathrm{R}$ & 1 & $A$ & $S$ & $E$ & $C$ \\
\hline \multirow{6}{*}{ 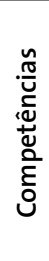 } & $\mathrm{R}$ & 1 & $0,491^{* *}$ & $0,257^{* *}$ & 0,145 & $0,410^{* *}$ & $0,451^{* *}$ \\
\hline & $\mathrm{I}$ & $0,491^{* *}$ & 1 & $0,567^{* *}$ & $0,440^{* *}$ & $0,457^{* *}$ & $0,504^{* *}$ \\
\hline & A & $0,257^{* *}$ & $0,567^{* *}$ & 1 & $0,509^{* *}$ & $0,375^{* *}$ & $0,492^{* *}$ \\
\hline & $\mathrm{S}$ & 0,145 & $0,440^{* *}$ & $0,509^{* *}$ & 1 & $0,469^{* *}$ & $0,441^{* *}$ \\
\hline & $E$ & $0,410^{* *}$ & $0,457^{* *}$ & $0,375^{* *}$ & $0,469^{* *}$ & 1 & $0,469^{* *}$ \\
\hline & $C$ & $0,451^{* *}$ & $0,504^{* *}$ & $0,492^{* *}$ & $0,441^{* *}$ & $0,469^{* *}$ & 1 \\
\hline
\end{tabular}

${ }^{*} \mathrm{p}<0,05{ }^{* *} \mathrm{p}<0,01$

Quadro 6. Correlações das competências percebidas entre os tipos RIASEC obtidas no $9^{\circ}$ ano

\begin{tabular}{|c|c|c|c|c|c|c|c|}
\hline \multirow{2}{*}{\multicolumn{2}{|c|}{$9^{\circ}$ ano }} & \multicolumn{6}{|c|}{ Competências } \\
\hline & & $\mathrm{R}$ & I & A & $S$ & $E$ & C \\
\hline \multirow{6}{*}{ 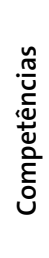 } & $\mathrm{R}$ & 1 & $0,444^{* *}$ & $0,163^{*}$ & 0,002 & $0,246^{* *}$ & $0,316^{* *}$ \\
\hline & I & $0,444^{* *}$ & 1 & $0,417^{* *}$ & $0,358^{* *}$ & $0,232^{* *}$ & $0,335^{* *}$ \\
\hline & A & $0,163^{* *}$ & $0,417^{* *}$ & 1 & $0,543^{* *}$ & $0,442^{* *}$ & $0,346^{* *}$ \\
\hline & $\mathrm{S}$ & 0,002 & $0,358^{* *}$ & $0,543^{* *}$ & 1 & $0,439^{* *}$ & $0,416^{* *}$ \\
\hline & $\mathrm{E}$ & $0,246^{* *}$ & $0,232^{* *}$ & $0,442^{* *}$ & $0,439^{* *}$ & 1 & $0,385^{* *}$ \\
\hline & C & $0,316^{* *}$ & $0,335^{* *}$ & $0,346^{* *}$ & $0,416^{* *}$ & $0,385^{* *}$ & 1 \\
\hline
\end{tabular}

${ }^{*} \mathrm{p}<0,05^{* *} \mathrm{p}<0,01$ 
Em todos os anos de escolaridade as competências de tipo Realista estão mais fortemente associadas às de tipo Investigador e as competências de tipo Artístico mais associadas às de tipo Social. Nos $3^{\circ}$ e $9^{\circ}$ anos as competências de tipo Empreendedor estão mais fortemente associadas às competências de tipo Artístico. Nos $3^{\circ}$ e $6^{\circ}$ anos de escolaridade as competências de tipo Convencional apresentam correlações mais elevadas com as competências de tipo Investigador.

Quer ao nível dos interesses, quer das competências percebidas, o tipo Realista associa-se mais fortemente ao tipo Investigador e o tipo Artístico ao Social, em todos os três anos de escolaridade. Tal como Holland previa, as associações entre tipos parecem ser mais fortes quanto mais próximos eles se situam no modelo hexagonal.

Por fim, três análises independentes de escalonamento multidimensional não métrico foram executadas recorrendo ao modelo Euclidiano MDS (ALSCAL). Na Figura 1 apresentam-se graficamente as configurações dos tipos de Holland (mapas MDS), nas duas dimensões especificadas a priori, para cada uma das sub-amostras de participantes neste estudo (alunos dos $3^{\circ}, 6^{\circ}$ e $9^{\circ}$ anos de escolaridade). Note-se que efectuámos uma transposição das coordenadas do gráfico para que a representação visual correspondesse à ordem R-I-A-S-E-C habitual (disposição dos tipos sequencialmente no sentido dos ponteiros do relógio).

Analisando os três mapas reproduzidos na Figura 1 constata-se que os pontos (os seis tipos de Holland) não reproduzem, senão muito imperfeitamente, a configuração de tipo circular teoricamente esperada. Pode-se, no entanto, constatar uma crescente aproximação a este padrão espacial em função da idade/escolarização dos participantes. Na amostra de alunos mais novos ( $3^{\circ}$ ano do ensino básico) verifica-se uma primeira inversão na ordem de $\mathrm{R}$ e $\mathrm{C}$, este último aparecendo mais próximo de I do que seria previsto. Há ainda uma segunda inversão entre A e S.

Todavia, há alguma evidência acerca das polaridades pressupostas no modelo dos tipos de personalidade de Holland (por exemplo, o mapa revela que o tipo Realista está suficientemente distanciado do tipo Social). As medidas do grau de ajustamento para este primeiro modelo são boas. O programa precisou apenas de quatro iterações para atingir uma solução, sendo o valor de S-Stress = 0,01, para a solução final. Por sua vez, o índice de stress (fórmula de Kruskal) = 0,035 e o $R^{2}=0,994$. 
(a) $3^{\circ}$ ano de escolaridade

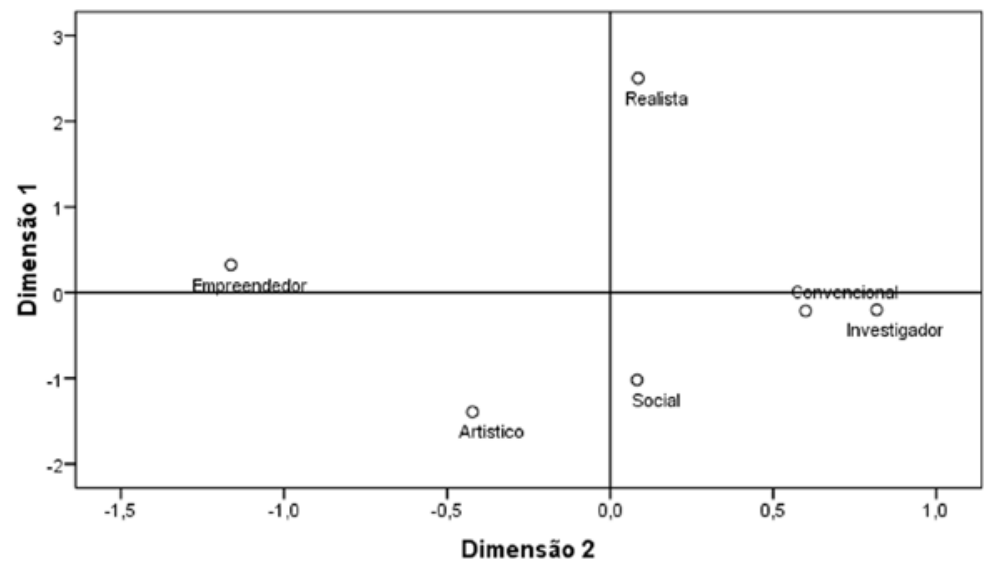

(b) $6^{\circ}$ ano de escolaridade

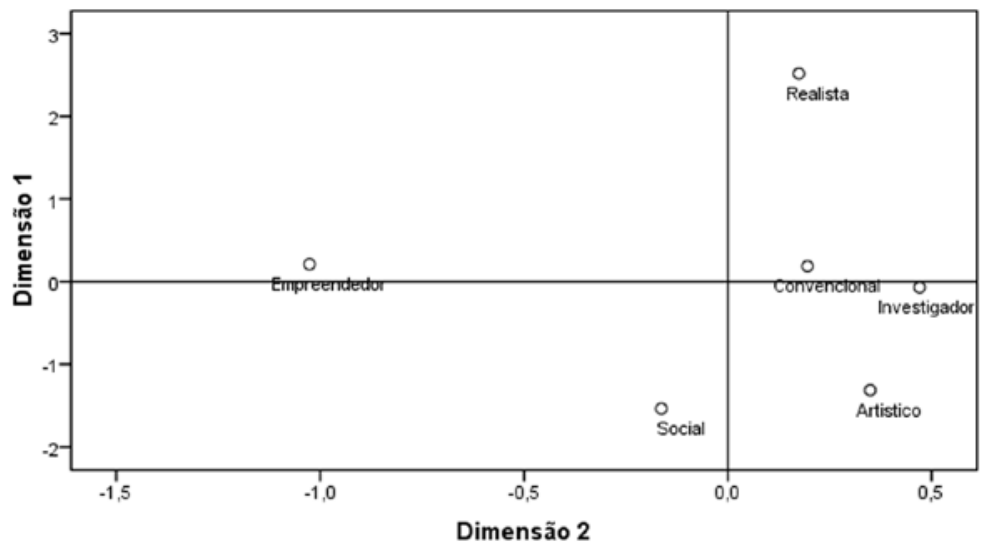

(c) $9^{\circ}$ ano de escolaridade

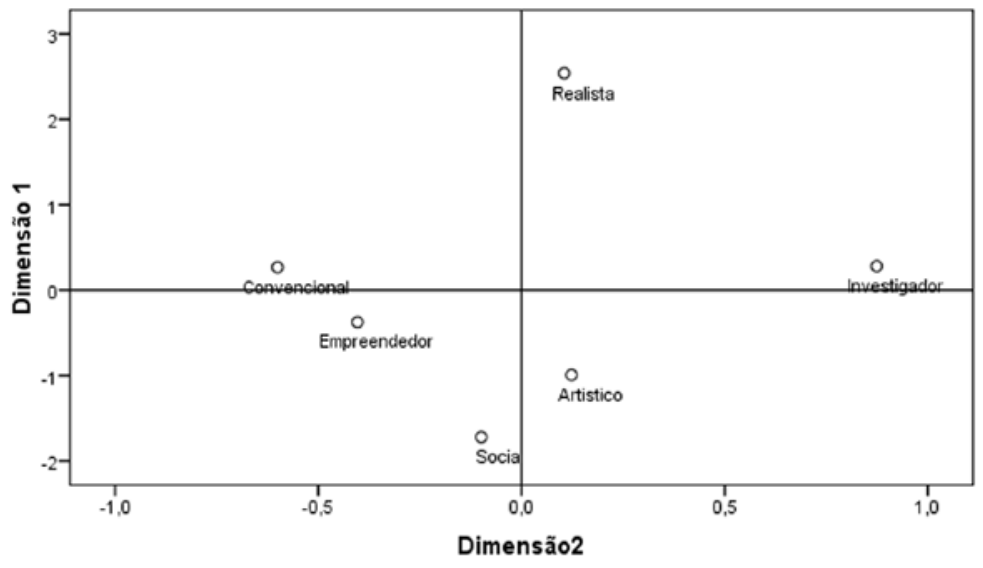

Figura 1. Gráficos MDS (Mapas) para as amostras do $3^{\circ}, 6^{\circ}$ e $9^{\circ}$ anos de escolaridade 
Analisando o mapa MDS para a amostra de participantes do $6^{\circ}$ ano de escolaridade (Fig. 1 [b]) chegamos a uma descrição similar à já referida para a primeira amostra. No caso presente, porém, apenas encontramos uma inversão na ordem esperada entre os tipos, designadamente entre R e C. Os indicadores do grau de ajuste, para este segundo modelo, são também bons, embora o programa tenha necessitado de 10 iterações para encontrar uma solução final, tendo-se obtido os valores de S-Stress, stress e $R^{2}$, de 0,005, 0,016 e 0,999, respectivamente.

$\mathrm{Na}$ amostra de alunos do $9^{\circ}$ ano de escolaridade existe, comparativamente às análises precedentes, uma maior aproximação dos tipos à representação circular teoricamente esperada, apesar de subsistirem fortes discrepâncias dos dados observados relativamente a esta forma geométrica. Em todo o caso, os indicadores do grau de ajuste para este modelo são bastante bons (S-Stress = 0,007; stress $\left.=0,013 ; R^{2}=1\right)$.

\section{Conclusão}

Os resultados obtidos através do ICA-R vão no sentido de que são as crianças mais novas que têm pontuações mais elevadas em todos os indicadores de interesses e de percepção de competências. Esta tendência poderá estar relacionada com o facto de as crianças mais novas ainda não serem capazes de discriminar com precisão os seus interesses e competências percebidas relativamente a domínios de actividade mais específicos. Segundo Osipow (1983), à medida que a idade avança as crianças avaliam-se a si mesmas, bem como ao ambiente que as rodeia, de modo mais realista (mais consentâneo com a realidade), daí que o decréscimo nas pontuações, tanto ao nível dos interesses como das competências percebidas, que verificámos à medida que aumentava o nível de escolaridade, possa estar relacionado com a capacidade de discriminarem, de modo mais realista, aquilo que gostam daquilo que não gostam de fazer, bem como as actividades que são capazes de desempenhar bem e aquelas em que revelam mais dificuldades. Talvez seja esta a principal razão pela qual os alunos mais novos, de um modo geral, obtenham resultados elevados e indiferenciados ao nível das competências, também verificados noutros estudos (Harter, 1982), e à medida que a idade aumenta regista-se um decréscimo nas pontuações nos interesses e nas competências percebidas (Tracey, 2002).

Presentemente, no domínio da Psicologia Vocacional, podemos recorrer a um número significativo de trabalhos sobre a estrutura dos interesses vocacionais (e.g., Rounds \& Day, 1999). Estas análises, porém, são muito mais infrequentes nas camadas mais jovens da população e, em particular, das que se encontram no período da infância 
(e.g., Tracey, 2001). A maioria dos autores, no entanto, considera que esta lacuna no nosso conhecimento tem de ser rapidamente preenchida se tivermos como objectivo alcançar uma visão compreensiva do desenvolvimento psicológico ao longo da vida. $\mathrm{O}$ estudo das percepções de auto-eficácia de carreira, ou das percepções de competências na infância, iniciou-se posteriormente ao dos interesses, encontrando-se, por esse motivo, num estádio de desenvolvimento mais preliminar e incipiente. Neste estudo pretendemos contribuir para o aprofundamento do conhecimento existente acerca da estrutura, ou dimensionalidade, das percepções de competências de crianças e jovens, através da aplicação do procedimento de escalonamento multidimensional (MDS) a dados recolhidos com a versão Portuguesa do ICA-R. Neste trabalho usámos uma abordagem exclusivamente exploratória das matrizes de dissemelhanças entre os seis tipos de personalidade de Holland, obtidas a partir das percepções de competências dos participantes das três sub-amostras estudadas (alunos dos $3^{\circ}$, $6^{\circ}$ e $9^{\circ}$ anos de escolaridade). As investigações realizadas nos EUA (e.g., Tracey \& Ward, 1998), concluíram que há uma relação positiva entre o ajustamento do modelo circular e a idade e que, adicionalmente, as amostras de alunos do ensino básico, comparativamente aos alunos do ensino superior, tendem a avaliar os interesses e as competências usando dimensões distintas. Os resultados desta investigação corroboram directamente a primeira conclusão referida e dão algum apoio à segunda asserção. Ainda que a representação circular da percepção de competências RIASEC apareça de uma forma muito vaga nos três mapas perceptuais que apresentámos, essa estrutura, porém, emerge de um modo mais claro nos resultados dos alunos do $9^{\circ}$ ano do que nas matrizes produzidas pelos seus colegas mais novos. A esta conclusão deve-se atribuir um carácter fundamentalmente heurístico e provisório, uma vez que não realizámos qualquer teste formal, de tipo confirmatório, do ajustamento dos dados ao modelo "circumplexo." (e.g, Tracey, 2000). Determinar as dimensões subjacentes às percepções de competências dos tipos de Holland não foi um objectivo deste estudo, por isso os comentários que se seguem podem considerar-se, no estado actual da investigação, como especulativos e transitórios. Embora a especificação do número de dimensões (neste caso duas) tenha sido efectuada a priori com base na literatura existente sobre esta questão, a verdade é que empiricamente duas dimensões parecem ser suficientes para representarem parcimoniosamente as relações existentes nas observações recolhidas. Determinar o significado psicológico de cada uma destas dimensões é matéria que deixamos para as próximas investigações, todavia, o modelo bidimensional Pessoas-Coisas, Ideias-Dados, proposto por Prediger (1982), poderá constituir uma boa ferramenta conceptual para abordarmos esta questão (note-se que a dimensão 2, representada nos mapas MDS da Fig. 1, tem alguma correspondência com a polaridade Pessoas [tipo S] vs. Coisas [tipo R]). As próximas investigações deverão testar esta hipótese interpretativa usando, por exemplo, processos de validação externa concomitantemente com os procedimentos MDS clássicos. 
Apesar de termos centrado as nossas análises na variável ano de escolaridade, a revisão da literatura indica-nos diversos factores influentes no desenvolvimento vocacional e, consequentemente, nas percepções de competência pelas crianças, por exemplo, o género (Betz, Borgen, \& Harmon, 1996; Tracey \& Ward, 1998; Tracey, 2002; David, 2007) ou mesmo o rendimento escolar (David, 2007). Estas dimensões também merecem ser alvo da atenção em futuras investigações. Em conclusão, os resultados que obtivemos neste estudo exploratório parecem-nos reunir bastante interesse não só do ponto de vista teórico como ainda no plano prático, especialmente no que diz respeito ao planeamento e à implementação de intervenções precoces de cariz vocacional.

\section{Referências bibliográficas}

Auger, R., Blackhurst, A., \& Wahl, K. (2005). The development of elementary-aged children's career aspirations and expectation. Professional School Counseling, 8, 322-329.

Bandura, A. (1977). Towards a unifying theory of behavior change. Psychological Review, 84 , 199-215.

Bandura, A. (1982). Self-efficacy mechanism in human agency. American Psychologist, 37, 122-147. Barak, A. (1981). Vocational interests: a cognitive view. Journal of Vocational Behavior, 19, 1-14. Betsworth, D. G., \& Fouad, N. A. (1997). Vocational interests: A look at the past 70 years and a glance at the future. Career Development Quarterly, 46, 23-47.

Betz, N. E., \& Hackett, G. (1981). The relationship of career related self-efficacy expectations to perceived career options in college women and men. Journal of Counseling Psychology, 28, 399-410.

Betz, N. E., Harmon, L. W., \& Borgen, F. H. (1996). The relationships of self-efficacy for the Holland themes to gender, occupational group membership and vocational interests. Journal of Counseling Psychology, 43, 90-98.

Boyle G. J., \& Fabris, S. (1992). LISREL analysis of the RIASEC model: Confirmatory and congeneric factor analysis of Holland's Self-Directed Search. Personality and Individual Differences, 13, 1077-1084.

Brown, D. (2003). Career Information, Career Counseling and Career Development ( $8^{\text {th }}$ ed.). Boston: Allyn and Bacon.

David, R. (2007). O Desenvolvimento Vocacional na Infância: Um Estudo Exploratório com Alunos do Ensino Básico. Dissertação de Mestrado em Psicologia da Motivação apresentada à Faculdade de Psicologia e Ciências da Educação da Universidade de Coimbra.

Dweck, C. S., \& Elliott, E. S. (1983). Achievement motivation. In P. H. Mussen (Gen. Ed.) \& E. M. Hetherington (Vol. Ed.), Handbook of child psychology: vol IV. Social and personality development (pp. 643-691). New York: Wiley.

Donnay, D., \& Borgen, F. (1999). The incremental validity of vocational self-efficacy: an examination of interest, self-efficacy and occupation. Journal of Counseling Psychology, 46 (4), 432-447. 
Everitt, B. S., \& Dunn, G. (2001). Applied multivariate data analysis (2nd ed.). London: Hodder Arnold.

Ferreira, J. A., Santos, E. J., Fonseca, A. C., \& Haase, R. F. (2007). Early predictors of career development: A 10-year follow-up study. Journal of Vocational Behavior, 70, 61-77.

Hair, J. F., Tatham, R. L., Anderson, R. E., \& Black, W. (1998). Multivariate data analysis (5th ed.). Upper Saddle River, NJ: Prentice Hall.

Harter, S. (1982). The perceived competence scale for children. Child Development, 53, 87-97. Hartung, P. J., Porfeli, E. J., \& Vondracek, F. W. (2005). Child vocational development: A review and reconsideration. Journal of Vocational Behavior, 66, 385-419.

Helwing, A. A. (2003). The measurement of Holland types in a 10-year longitudinal study of a sample of students. Journal of Employment Counseling, 40, 24-32.

Holland, J. L. (1973). Making vocational choices: A theory of careers. Englewood Cliffs, NJ: Prentice Hall.

Holland, J. L. (1985). Making vocational choices: A theory of vocational personalities and work environments ( $2^{\text {nd }}$ ed.). Englewood Cliffs, NJ: Prentice Hall.

Holub, S. C. (2005). Young children's perceived competence: validation of a new instrument and ecological influences on overly positive self-perceptions in prescholers. Bowling Green State University.

Leitão, L. M., \& Miguel, J. P. (2001). Os interesses revisitados. Psychologica, 26, 79-104.

Lent, R. W., Brown, S. D., \& Hackett, G. (1994). Toward a unifying social cognitive theory of career and academic interest, choice and performance. Journal of Vocational Behavior, 45, 79-122.

Lent, R. W., Brown, S. D., \& Hackett, G. (2002). Social Cognitive Career Theory. In D. Brown (Ed.), Career choice and development (4 ${ }^{\text {th }}$ ed., pp. 255-311). San Francisco: Jossey-Bass.

Lent, R. W., Tracey, T., Brown, S., Soresi, S., \& Nota, L. (2006). Development of Interests and Competency Beliefs in Italian Adolescents: An Exploration of Circumplex Structure and Bidirectional Relationships. Journal of Counseling Psychology, 53, 181-191.

Lopez, F. G., Lent, R. W., Brown, S. D., \& Gore, P. A. (1997). Role of social-cognitive expectations in high school student's mathematics-related interest and performance. Journal of Counseling Psychology, 44, 44-52.

Osipow, S. H. (1983). Theories of career development (3 ${ }^{\text {rd }}$ ed.). Englewood Cliffs, NJ: Prentice Hall.

Paixão, M. P., \& Silva, J.T. (2001). Estudo do funcionamento motivacional em momentos críticos de tomada de decisão vocacional: Estudo exploratório. Psychologica, 26, 175-185.

Parsons, J. E., \& Ruble, D. N. (1977) The development of achievement-related expectancies. Child Development, 48, 1075-1079.

Pelletier, D., Noiseaux, G., \& Bujold, C. (1982). Desenvolvimento vocacional e crescimento pessoal : enfoque operatório (trad.). Petróplis: Vozes.

Prediger, D. J. (1982). Dimensions underlying Holland's hexagon: Missing link between interests and occupations? Journal of Vocational Behavior, 21, 259-287.

Rounds, J., \& Day, S. (1999). Describing, evaluating, and creating vocational interests structures. In M. L. Savickas \& A. R. Spokane (Eds.), Vocational interests: Meaning, measurement and counseling use (pp. 103-133). Palo Alto, CA: Davies-Black Publishing.

Savickas, M. L. (1999). The psychology of interests. In: M. L. Savickas \& A. R. Spokane (Eds.), Vocational interests: Meaning, measurement, and counseling use (pp. 19-56). Palo Alto, CA: Davies-Black Publishing. 
Simões, M. R. (1994). Investigações no âmbito da Aferição Nacional do Teste das Matrizes Progressivas Coloridas de Raven (M.P.C.R.). Dissertação de Doutoramento em Psicologia, especialização em Avaliação Psicológica, apresentada à Faculdade de Psicologia e de Ciências da Educação da Universidade de Coimbra.

Sollerhed, A., Apitzsch, E., Råstam, L., \& Ejlertsson, G. (2008). Factors associated with young children's self-perceived physical competence and self-reported physical activity. Health Education Research, 23, 125-136.

Super, D. (1990). A life-span, life space approach to career development. In Brown \& Brooks (Eds.), Career Choice and Development ( $2^{\text {nd }}$ ed., pp. 197-261). San Francisco: Jossey-Bass Publishers.

Takane, Y., Young, F. W., \& de Leeuw, J. (1977). Nonmetric individual differences multidimensional scaling: An alternating least squares method with optimal scaling features. Psychometrika, 42, 7-67.

Taveira, M. C. (2004) (Ed.). Desenvolvimento Vocacional ao Longo da Vida: Fundamentos, princípios e orientações. Coimbra: Almedina.

Tracey, T. G. (1997). The structure of interests and self-efficacy estimations: An expanded examination of the spherical model of interests. Journal of Counseling Psychology, 44, 32-43.

Tracey, T. G. (2000). Analysis of circumplex models. In H. E. Tinsley \& S. D. Brown (Eds.), Handbook of applied multivariate statistics and mathematical modeling (pp. 641-664). San Diego, CA: Academic Press.

Tracey, T. G. (2001). The development of structure of interests in children: Setting the stage. Journal of Vocational Behavior, 59, 89-104.

Tracey, T. (2002). Development of Interests and Competency Beliefs: A 1-year longitudinal study of fifth-to-right-grade students, using the ICA-R and Structural Equation Modeling. Journal of Counseling Psychology, 49, 148-163.

Tracey, T. G., \& Sodano, S. S. (2008) Issues of Stability and Change in Interest Development. The Career Development Quarterly, 57, 51-62.

Tracey, T. G., \& Ward, C. C. (1998). The structure of children's interests and competencies perceptions. Journal of Counseling Psychology, 45, 290-303.

Trice, A. D. (1991). Stability of children's career aspirations. Journal of Genetic Psychology, 152, 137-139.

Young, F. W., \& Harris, D. F. (2007). Multidimensional scaling. In M. J. Norŭsis (Ed.), SPSS 15.0 Advanced statistical procedures companion (pp. 335-404). Upper Saddle River, NJ: Prentice Hall.

Wahl, K. H., \& Blackhurst, A. (2000). Factors affecting the Occupational and Educational Aspirations of Children and Adolescents. Professional School Counseling, 3, 367-374.

Weinrach, S., \& Srebalus, D. (1990). Holland's Theory of Careers. In D. Brown \& L. Brooks (Eds.), Career Choice and Development ( $2^{\text {nd }}$ ed., pp. 37-67). San Francisco: Jossey-Bass. 


\section{Intérêts et compétences perçues dans des enfants de l'enseignement basique}

Malgré de l'enfance être considérée comme la base du développement vocationnelle, peu sont les études développées dans cette bande étaire concernant les variables impliquées et le papier que lequel jouent dans ce processus. En prétendant colmater certaines de ces lacunes nous avons cherché à explorer quelques caractéristiques structurelles et corrélez des intérêts et des compétences perçues d'enfants de l'enseignement basique $\left(3^{\circ}, 6^{\circ}\right.$ et $9^{\circ}$ années de scolarité), en faisant appel, à cet effet, à la traduction et à l'adaptation portugaise de l' ICA - R de Tracey et de Ward, qui a dans considération les six types de personnalité énoncés par Holland (R-I-A-S-E-C).

MOTS-CLÉS: Développement vocationnelle; Enfance; Compétences perçues; Intérêts.

\section{Interests and perceived competencies in elementary and middle school children}

Although childhood is generally considered as the basis of the vocational development process, few are the studies carried out in this developmental phase which try to point out the variables involved and what role they develop in this process. While trying to overcome some of the gaps above mentioned, in this study we explored some of the structural and correlational characteristics of the interests and the perceived competencies of children attending basic school $\left(3^{\text {rd }}, 6^{\text {th }}\right.$ and $9^{\text {th }}$ grades, respectively). In order to achieve that goal, we translated and adapted the Portuguese version of Tracey and Ward's ICA-R, which contemplates the six vocational personality types identified by Holland (R-I-A-S-E-C).

KEY-WORDS: Vocational development; Childhood; Perceived competencies; Interests. 